\title{
Nonlinear feedback controller for the synchronization of hyper (chaotic) systems with known parameters
}

\author{
Muhammad Haris ${ }^{\mathrm{a}, \mathrm{c}, *}$, Muhammad Shafiq ${ }^{\mathrm{b}}$, Adyda Ibrahim $^{\mathrm{c}}$, Masnita Misiran ${ }^{\mathrm{c}}$ \\ ${ }^{a}$ Center of Foundation Studies, University of Buraimi, Al Buraimi, Oman. \\ ${ }^{b}$ Department of Electrical and Computer Engineering, Sultan Qaboos University, Muscat, Oman. \\ ${ }^{c}$ Department of Mathematics and Statistics, School of Quantitative Sciences, Universiti Utara Malaysia, 06010, Kedah, Malaysia.
}

\begin{abstract}
This paper proposes, designs, and analyses a novel nonlinear feedback controller that realizes fast, and oscillation free convergence of the synchronization error to the equilibrium point. Oscillation free convergence lowers the failure chances of a closed-loop system due to the reduced chattering phenomenon in the actuator motion, which is a consequence of low energy sm ooth control signal. The proposed controller has a novel structure. This controller does not cancel nonlinear terms of the plant in the closed-loop; this attribute improves the robustness of the loop. The controller consists of linear and nonlinear parts; each part executes a specific task. The linear term in the controller keeps the closed-loop stable, while the nonlinear part of the controller facilitates the fast convergence of the error signal to the vicinity of the origin. Then the linear controller synthesizes a smooth control signal that moves the error signals to zero without oscillations. The nonlinear term of the controller does not contribute to this synthesis. The collaborative combination of linear and nonlinear controllers that drive the synchronization errors to zero is innovative. The paper establishes proof of global stability and convergence behavior by describing a detailed analysis based on the Lyapunov stability theory. Computer simulation results of two numerical examples verify the performance of the proposed controller approach. The paper also provides a comparative study with state-of-the-art controllers.
\end{abstract}

Keywords: Chaotic system, synchronization, Lyapunov stability, nonlinear feedback controller.

2020 MSC: 34H10, 34H05, 34D06.

(C)2021 All rights reserved.

\section{Introduction}

The terminology chaos synchronization represents the tracking of oscillatory trajectories of two or more chaotic systems. Chaotic systems are vulnerable to the initial conditions and can produce singularities in the system. The response of such systems is random, unpredictable, and complex. Pecora and Carroll introduced the notion of chaos synchronization [18], as a consequence of the successful development of an experiment for the synchronization of Rossler and Lorenz chaotic systems using a feedback

\footnotetext{
*Corresponding author

Email addresses: hariskhan4u@yahoo.com (Muhammad Haris), shafiqsaeeda@yahoo.com, mshafiq@squ.edu.om (Muhammad Shafiq), adyda@uum.edu.my (Adyda Ibrahim), masnita@uum.edu.my (Masnita Misiran)
}

doi: $10.22436 /$ jmcs.023.02.05

Received: 2020-03-13 Revised: 2020-06-03 Accepted: 2020-08-17 
Table 1: Notation and symbols.

\begin{tabular}{|l|l|}
\hline Symbols & Description \\
\hline$T$ & Transpose of a vector (matrix) \\
\hline$R^{n}$ & $n$-dimensional Euclidean space \\
\hline$R^{m}$ & m-dimensional Euclidean space \\
\hline$u(t)=\left[u_{1}(t), u_{2}(t), \ldots, u_{n}(t)\right]^{\top} \in R^{n \times 1}$ & Control input vector \\
\hline$\chi^{m}(t)=\left[\chi_{1}^{m}(t), \chi_{2}^{m}(t), \ldots, \chi_{n}^{m}(t)\right]^{\top} \in R^{n \times 1}$ & State vectors of the master system \\
\hline$\chi^{s}(t)=\left[\chi_{1}^{s}(t), \chi_{2}^{s}(t), \ldots, \chi_{n}^{s}(t)\right]^{\top} \in R^{n \times 1}$ & State vectors of the slave system \\
\hline$F\left(\chi^{m}(t)\right)=\left[f_{1}\left(\chi^{m}(t)\right), f_{2}\left(\chi^{m}(t)\right), \ldots, f_{n}\left(\chi^{m}(t)\right]^{\top} \in R^{n \times 1}\right.$ & $\begin{array}{l}\text { The nonlinear continuous-time function of the state-vector } \\
\text { for the master system }\end{array}$ \\
\hline$F\left(\chi^{s}(t)\right)=\left[f_{1}\left(\chi^{s}(t)\right), f_{2}\left(\chi^{s}(t)\right), \ldots, f_{n}\left(\chi^{s}(t)\right]^{\top} \in R^{n \times 1}\right.$ & $\begin{array}{l}\text { The nonlinear continuous-time function of the state-vector } \\
\text { for the slave system }\end{array}$ \\
\hline$F(\chi(t)): R^{n \times 1} \times R^{n \times 1} \rightarrow R^{n \times 1}$ & Nonlinear smooth continuous function \\
\hline$\Xi(t)=\chi^{m}(t)-\chi^{s}(t) \in R^{n \times 1}$ & The state-error vector of master and slave systems \\
\hline$a=\left[a_{1}, a_{2}, \ldots, \alpha_{n}\right]^{T} \in R^{n \times 1}$ & Parameters vector of the master system \\
\hline$b=\left[b_{1}, b_{2}, \ldots, b_{n}\right]^{\top} \in R^{n \times 1}$ & Parameters vector of the slave system \\
\hline$A \in R^{n \times n}$ & $n \times n$ matrix consisting of elements from $a$ \\
\hline$B \in R^{n \times n}$ & $n \times n$ matrix consisting of elements from $b$ \\
\hline
\end{tabular}

control algorithm. Since then, researchers show immense interest in studying the synchronization of chaotic systems and developing controllers due to their widespread applications.

Tracking trajectories is one of the control strategies that realizes the synchronization of chaotic systems. These control techniques include the nonlinear control (NLC) methods [8,11], finite-time control strategies (FTS) $[5,23,26]$ active control approach [1], adaptive control techniques [3, 21], sliding mode controllers $[9,15]$, quantized control algorithms [29], switching control strategies [20], backstopping control methods [22], intermittent control scheme [28], and the fuzzy control techniques [19], among others.

The synchronization of chaotic systems has successful applications in natural science and engineering, such as secure communication systems [4], neural network systems [26], aerospace technology [10], and chemical reactors [13], etc. Extensive applications of chaotic synchronization have motivated many researchers to study and develop efficient algorithms. [11] proposes a nonlinear control strategy and investigates the synchronization of two identical and two non-identical chaotic systems. Based on the Lyapunov stability theory [11], the authors design a nonlinear state-feedback controller to cancel all nonlinear terms of the plant and synchronize two identical Lu chaotic systems and two non-identical Lur'e-like system and Genesio system. Similarly, [17] achieves synchronization for two identical chaotic systems based on the Lyapunov stability theory. It improves the performance in terms of the oscillation amplitude and rate of the convergence error to the steady-state. Reduction in energy consumption is another challenge in the controller design. [30] proposes a methodology that achieves better energy utilization than [17], and shows that the closed-loop is exponential stable. [27] develops an FTS controller for synchronization of unified chaotic systems with uncertainties and use the Lyapunov theory to prove the stability. [24] investigates the NLC strategy for the synchronization and anti-synchronization of Lu chaotic systems and Bhalekar-Gejji chaotic system. To reduce the oscillation amplitude of the error signals, [25] applies a single input sliding mode control that accomplishes synchronization between uncertain Lu identical chaotic (hyperchaotic) systems. [6] introduces an NLC technique that investigates synchronization between two non-identical hyperchaotic systems.

Most of the above synchronization control strategies eliminate the nonlinear terms in the closed-loop system. The presence of the nonlinear terms in the closed-loop enhances the speed of convergence and strengthens the security of the information signal in secure communication systems [14]. Therefore, it is challenging to retain the nonlinear terms and show the closed-loop system stability in the synchronization of chaotic systems [2]. One of the core issues in the synchronization of chaotic systems is the slow rate of convergence [16]. The synchronization error with large oscillations is another problem that creates chattering and makes the system noisy. Penalization of these oscillations by incorporating nonlinear 
cancellation terms in the controller synthesizes substantial control effort, which consumes high energy [7].

The above discussion poses a challenge to design an energy-efficient controller for quality synchronization. Quality synchronization refers to the fast and oscillation free trajectories in the steady-state.

This paper proposes an NLC feedback controller that expedites the convergence of the synchronization error without eliminating nonlinear terms in the closed-loop. The nonlinear part of the proposed controller decreases the amplitude of oscillations in the error signals and increases the pace of synchronization convergence. The linear part keeps the closed-loop system stable; it assures the convergence of the state error to zero. The proposed controller synthesizes an adequate control signal to penalize the large errors rapidly.

The rest of the paper is composed as follows. Section 2 describes the challenge, and 3 proposes a generalized methodology for the synchronization of two identical chaotic(hyperchaotic) systems. Sections 4 and 5 present the numerical simulations and a comparative study for the synchronization of two identical chaotic Lu systems and two identical hyperchaotic Lu systems, respectively. The paper concludes in Section 6 with some remarks.

\section{Description of the challenge}

Consider the following master and slave systems arrangement:

$$
\begin{aligned}
\text { Master system: } & \dot{\chi}^{m}(t)=A \chi^{m}(t)+F\left(\chi^{m}(t)\right), \\
\text { Slave system: } & \dot{\chi}^{s}(t)=B \chi^{s}(t)+F\left(\chi^{s}(t)\right)+u(t) .
\end{aligned}
$$

Definition 2.1. Let $\Xi(t)=\chi^{s}(t)-\chi^{m}(t) \in R^{n \times n}$, then the error system for the master and slave systems (2.1)-(2.2) is defined as follows:

$$
\begin{aligned}
\dot{\Xi}(t) & =B \chi^{s}(t)-A \chi^{m}(t)+F\left(\chi^{s}(t)\right)-F\left(\chi^{m}(t)\right)+u(t) \\
& =B \chi^{s}(t)-B \chi^{m}(t)+A \chi^{s}(t)-A \chi^{m}(t)+F\left(\chi^{s}(t)\right)-F\left(\chi^{m}(t)\right)+B \chi^{m}(t)-A \chi^{m}(t)+u(t) \\
& =B \Xi(t)+A \Xi(t)+Q\left(\chi^{m}(t), \chi^{s}(t)\right) \Xi(t)+u(t) \\
& =C \Xi(t)+Q\left(\chi^{m}(t), \chi^{s}(t)\right) \Xi(t)+u(t),
\end{aligned}
$$

where $C=A+B$ is a coefficient matrix, $C \Xi(t)$ and

$$
\mathrm{Q}\left(\chi^{\mathrm{m}}(\mathrm{t}), \chi^{\mathrm{s}}(\mathrm{t})\right) \Xi(\mathrm{t})=\mathrm{B} \chi^{\mathrm{m}}(\mathrm{t})-\mathrm{A} \chi^{\mathrm{m}}(\mathrm{t})+\mathrm{F}\left(\chi^{\mathrm{s}}(\mathrm{t})\right)-\mathrm{F}\left(\chi^{\mathrm{m}}(\mathrm{t})\right)
$$

represent the linear and nonlinear parts of the error dynamics system.

Objective: The objective of this article is to design a controller that accomplishes synchronization between the master-slave systems described in (2.1) and (2.2) rapidly with reduced oscillations in the transient phase and suppressed oscillations in the steady-state. This paper proposes a novel controller that synthesizes a control signal $u(t)$ that keeps the closed-loop dynamical system (2.3) globally asymptotic stable at the origin. The global asymptotic stable closed-loop assures,

$$
\lim _{t \rightarrow \infty}\|\Xi(t)\|=0
$$

\section{Propose controller to address the challenge}

This section describes the structure of the proposed controller and proves the closed-loop stability. The analysis establishes the proof of the closed-loop stability based on Assumption 3.1. 
Assumption 3.1. The chaotic attractors evolve in a region $M \subset R^{n}$, because the chaotic master-slave systems (2.1) and (2.2) owe bounded trajectories. Therefore, the chaotic attractors of the error system in (2.3) do not leave or overlap this region. As a consequent, there exist positive constants $M_{\chi^{m}}, M_{\chi^{s}} \in R$ such that [7],

$$
\chi^{m}(t) \leqslant M_{\chi^{m}}, \text { and } \chi^{s}(t) \leqslant M_{\chi^{s}}
$$

Theorem 3.2. If the Assumption 3.1 is satisfied, then relationship (2.3) synthesizes a control signal $u(t)$ that accomplishes the objective.

$$
u(t)=-K\left(1-e^{-\rho|\Xi(t)|}\right) \tanh (\beta \Xi(t))-L \Xi(t),
$$

where the diagonal matrices $\mathrm{K} \in \mathrm{R}^{\mathrm{n} \times \mathrm{n}}$ and $\mathrm{L} \in \mathrm{R}^{\mathrm{n} \times \mathrm{n}}$ represent the feedback control gains, $|\tanh (\gamma \Xi(\mathrm{t}))| \leqslant 1$ $\forall \Xi(\mathrm{t}) \neq 0,|\tanh (\Xi(\mathrm{t}))|=0$, for $\Xi(\mathrm{t})=0$, and $\rho>0$ and $\gamma>0$ are two real positive constants.

Proof. Using (3.1) into (2.3) yields:

$$
\dot{\Xi}(\mathrm{t})=\mathrm{C} \Xi(\mathrm{t})+\mathrm{Q}\left(\chi^{\mathrm{m}}(\mathrm{t}), \chi^{\mathrm{s}}(\mathrm{t})\right) \Xi(\mathrm{t})-\mathrm{K}\left(1-\mathrm{e}^{-\rho|\Xi(\mathrm{t})|}\right) \tanh (\gamma \Xi(\mathrm{t}))-\mathrm{L} \Xi(\mathrm{t}) .
$$

Consider the following Lyapunov candidate function:

$$
\mathrm{V}(\Xi(\mathrm{t}))=\frac{1}{2}\left(\Xi^{\top}(\mathrm{t}) \Xi(\mathrm{t})\right) \geqslant 0
$$

Taking the time derivative of (3.3) and using (3.2) give:

$$
\begin{aligned}
\dot{V}(\Xi(t))= & \Xi^{\top}(t) C \Xi(t)+\Xi^{\top}(t) Q\left(x^{m}(t), \chi^{s}(t)\right)(t) \Xi(t) \\
& -\Xi^{\top}(t) K\left(1-e^{-\rho \mid \Xi((t) \mid)} \tanh (\gamma \Xi(t))-\Xi^{\top}(t) L \Xi(t)\right. \\
= & -\Xi^{\top}(t)(-C-Q+L) \Xi(t)-\Xi^{\top}(t) K\left(1-e^{-\rho \mid \Xi((t) \mid}\right) \tanh (\gamma \Xi(t)) \\
\leqslant & -\Xi^{\top}(t)\left(-C-M_{x}-M_{\chi^{s}}+\mathrm{L}\right) \Xi(t)-\Xi^{\top}(t) K\left(1-e^{-\rho \mid \Xi((t) \mid}\right) \tanh (\gamma \Xi(t)) .
\end{aligned}
$$

If $\left(-C-M_{\chi^{m}}-M_{\chi^{s}}+L\right)$ and $K$ are positive definite then $\dot{V}(\Xi(t)) \leqslant 0$.

\section{Numerical example-1}

This section presents the synchronization between two identical chaotic Lu systems.

\subsection{Dynamics of the unified Lu chaotic system}

Equation (4.1) gives the dynamics of the unified chaotic system.

$$
\left\{\begin{array}{l}
\dot{\chi}_{1}(t)=(25 \alpha+10)\left(\chi_{2}(t)-\chi_{1}(t)\right) \\
\dot{\chi}_{2}(t)=(28-35 \alpha)\left(x_{1}(t)-\chi_{1}(t) \chi_{3}(t)+(29 \alpha-1) \chi_{2}(t),\right. \\
\dot{\chi}_{3}(t)=\chi_{1}(t) \chi_{2}(t)-\frac{(8+\alpha) \chi_{3}(t)}{3}
\end{array}\right.
$$

where $\chi_{1}(t), \chi_{2}(t)$, and $\chi_{3}(t)$ are the state variables, and $\alpha \in[0,1]$ represents the parameter. The dynamic system (4.1) represents the $\mathrm{Lu}$ chaotic system for $0.8<\alpha \leqslant 1$. Figures 1 and 2 show the 2D and 3D dynamic behaviors of the $\mathrm{Lu}$ chaotic system. 


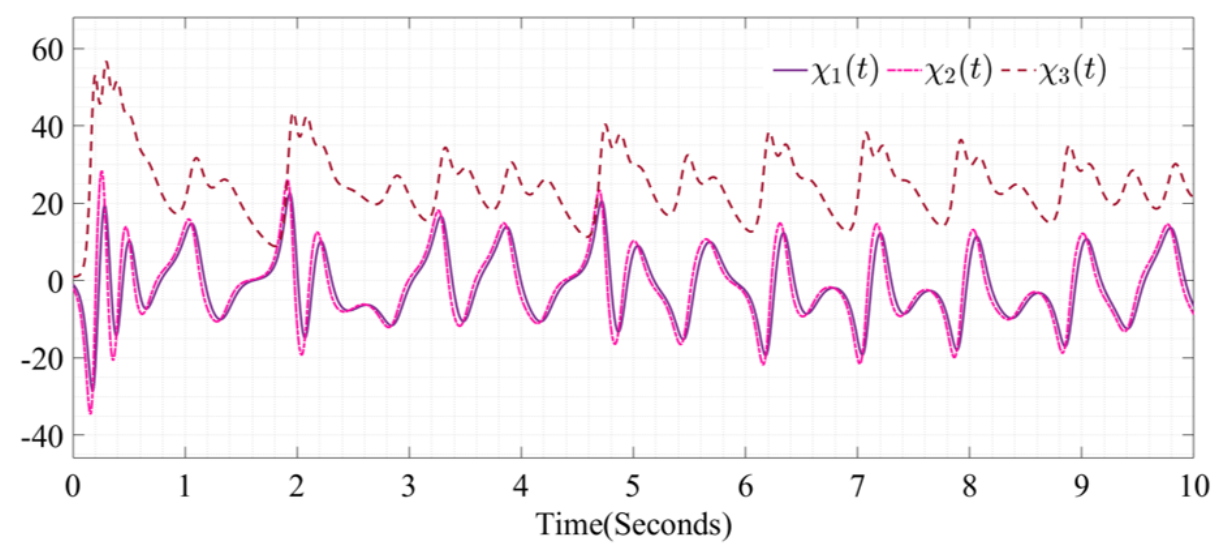

Figure 1: Behavior of the state variables.

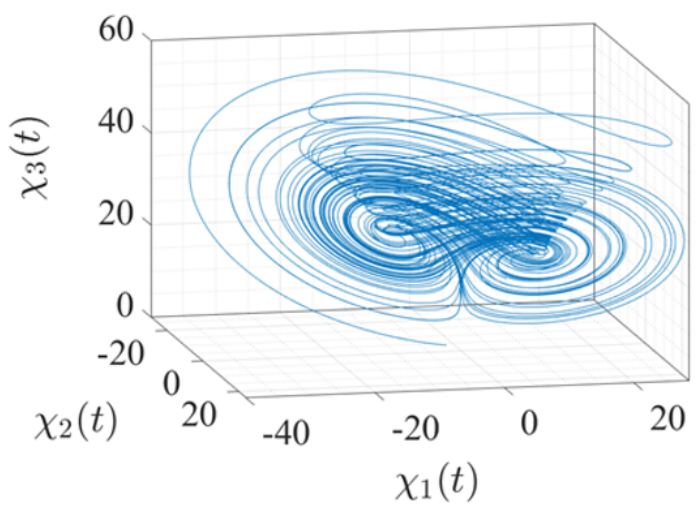

Figure 2: 3D phase portrait.

\subsection{Synchronization of two identical Lu chaotic systems}

This subsection presents a numerical example investigating the synchronization of two non-identical $\mathrm{Lu}$ chaotic systems using the master and slave systems synchronization organization. The state of the master system is denoted by $\chi^{m}(t)=\left[\chi_{1}^{m}(t), \chi_{2}^{m}(t), \chi_{3}^{m}(t)\right]^{\top}$, and the slave system is represented by $\chi^{s}(t)=\left[\chi_{1}^{s}(t), \chi_{2}^{s}(t), \chi_{3}^{s}(t)\right]^{\top}$. The master-slave system arrangement is given in (4.2) and (4.3).

$$
\begin{aligned}
& \text { Master system: }\left\{\begin{array}{l}
\dot{\chi}_{1}^{m}(t)=(25 \alpha+10)\left(\chi_{2}^{m}(t)-\chi_{1}^{m}(t)\right), \\
\dot{\chi}_{2}^{m}(t)=(28-35 \alpha)\left(\chi_{1}^{m}(t)-\chi_{1}(t) \chi_{3}^{m}(t)+(29 \alpha-1) \chi_{2}^{m}(t),\right. \\
\dot{\chi}_{3}^{m}(t)=\chi_{1}^{m}(t) \chi_{2}^{m}(t)-\frac{(8+\alpha) \chi_{3}^{m}(t)}{3},
\end{array}\right. \\
& \text { Slave system: }\left\{\begin{array}{l}
\dot{\chi}_{1}^{s}(t)=(25 \alpha+10)\left(\chi_{2}^{s}(t)-\chi_{1}^{s}(t)\right)+u_{1}(t), \\
\dot{\chi}_{2}^{s}(t)=(28-35 \alpha) \chi_{1}^{s}(t)-\chi_{1}^{s}(t) \chi_{3}^{s}(t)+(29 \alpha-1) \chi_{2}^{s}(t)+u_{2}(t), \\
\dot{\chi}_{3}^{s}(t)=\chi_{1}^{s}(t) \chi_{2}^{s}(t)-\frac{(8+\alpha) \chi_{3}^{s}(t)}{3}+u_{3}(t),
\end{array}\right.
\end{aligned}
$$

where $u(t)=\left[u_{1}(t), u_{2}(t), u_{3}(t)\right]$ is the control input vector. Let $\Xi_{i}(t)=\chi_{i}^{s}(t)-\chi_{i}^{m}(t), i=1,2,3$ denote the corresponding state error, then the error dynamical system for the master and slave systems (4.2) and (4.3) is given in (4.4).

$$
\left\{\begin{array}{l}
\dot{\Xi}_{1}(\mathrm{t})=(25 \alpha+10)\left(\Xi_{2}(\mathrm{t})-\Xi_{1}(\mathrm{t})\right)+\mathrm{u}_{1}(\mathrm{t}) \\
\dot{\Xi}_{2}(\mathrm{t})=(28-35 \alpha) \Xi_{1}(\mathrm{t})-\chi_{1}^{\mathrm{s}}(\mathrm{t}) \chi_{3}^{\mathrm{s}}(\mathrm{t})+\chi_{1}^{\mathrm{m}}(\mathrm{t}) \chi_{3}^{\mathrm{m}}(\mathrm{t})+(29 \alpha-1) \Xi_{2}(\mathrm{t})+\mathrm{u}_{2}(\mathrm{t}) \\
\dot{\Xi}_{3}(\mathrm{t})=\chi_{1}^{\mathrm{s}}(\mathrm{t}) \chi_{2}^{\mathrm{s}}(\mathrm{t})-\chi_{1}^{\mathrm{m}}(\mathrm{t}) \chi_{2}^{\mathrm{m}}(\mathrm{t})-\frac{(8+\alpha) \Xi_{3}(\mathrm{t})}{3}+\mathrm{u}_{3}(\mathrm{t})
\end{array}\right.
$$


It is easy to show that:

$$
\left\{\begin{array}{l}
\chi_{1}^{s}(t) \chi_{2}^{s}(t)-\chi_{1}^{m}(t) \chi_{2}^{m}(t)=\chi_{2}^{s}(t) \Xi_{1}(t)+\chi_{1}^{s}(t) \Xi_{2}(t)-\Xi_{1}(t) \Xi_{2}(t) \\
\chi_{1}^{s}(t) \chi_{3}^{s}(t)-\chi_{1}^{m}(t) \chi_{3}^{m}(t)=\chi_{3}^{s}(t) \Xi_{1}(t)+\chi_{1}^{s}(t) \Xi_{3}(t)-\Xi_{1}(t) \Xi_{3}(t)
\end{array}\right.
$$

Substituting (4.5) in (4.4) gives that:

$$
\left\{\begin{array}{l}
\dot{\Xi}_{1}(\mathrm{t})=(25 \alpha+10)\left(\Xi_{2}(\mathrm{t})-\Xi_{1}(\mathrm{t})\right)+\mathrm{u}_{1}(\mathrm{t}), \\
\dot{\Xi}_{2}(\mathrm{t})=(28-35 \alpha) \Xi_{1}(\mathrm{t})-\chi_{3}^{\mathrm{s}}(\mathrm{t}) \Xi_{1}(\mathrm{t})-\chi_{1}^{\mathrm{s}}(\mathrm{t}) \Xi_{3}(\mathrm{t})+\Xi_{1}(\mathrm{t}) \Xi_{3}(\mathrm{t})+(29 \alpha-1) \Xi_{2}(\mathrm{t})+\mathrm{u}_{2}(\mathrm{t}), \\
\dot{\Xi}_{3}(\mathrm{t})=\chi_{2}^{\mathrm{s}}(\mathrm{t}) \Xi_{1}(\mathrm{t})+\chi_{1}^{\mathrm{s}}(\mathrm{t}) \Xi_{2}(\mathrm{t})-\Xi_{1}(\mathrm{t}) \Xi_{2}(\mathrm{t})-\frac{(8+\alpha) \Xi_{3}(\mathrm{t})}{3}+\mathrm{u}_{3}(\mathrm{t}) .
\end{array}\right.
$$

\subsection{Numerical simulations and comparative study}

This subsection discusses the efficiency and performance of the proposed NLC (3.1). It provides comparative analysis in terms of synchronization time and amplitude of the oscillation with the state-of-the art feedback control laws (4.6) [17] and (4.7) [27]. The initial conditions for the master and slave systems (4.2) and (4.3) are set as $\left[\chi_{1}^{m}(0), \chi_{2}^{m}(0), \chi_{3}^{m}(0)\right]^{\top}=[-1,-2,1]^{\top}$, and $\left[\chi_{1}^{s}(0), \varnothing_{2}^{s}(0), \chi_{3}^{s}(0)\right]^{\top}=[4,-4,4]^{\top}$, respectively. This paper uses Simulink \& Matlab software in the 64-bit windows ten environment for all the numerical examples. The numerical solution of the dynamic systems is based on the fourth-order Runge-Kutta method with step size 0.001 .

The control signals $u_{i}(t), i=1,2,3$, given in (4.7) and (4.8) is synthesized by the feedback control strategy $[17,27]$, then these control signals in the error system (4.6) establish the synchronization of the master and slave systems (4.2) and (4.3).

The feedback control technique [17]: $\left\{\begin{array}{l}u_{1}(t)=-\left(\beta_{1}+\beta_{2}-z_{m}(t)\right) \Xi_{2}(t), \\ u_{2}(t)=-\left(\beta_{3}+k\right) \Xi_{2}(t), \\ u_{3}(t)=-y_{m}(t) \Xi_{2}(t),\end{array}\right.$
The feedback control technique [27]: $\left\{\begin{array}{l}u_{1}(t)=-(25+10 \alpha) e_{1}(t)+e_{1}^{\beta}(t), \\ u_{2}(t)=-K e_{2}(t)-e_{2}^{\beta}(t), \\ u_{3}(t)=-e_{3}^{\beta}(t) .\end{array}\right.$

Table 2 summarizes the comparative analysis of the simulation results. Results in Figure 3 (a)-(c) illustrate that the proposed feedback controller (3.1) accomplishes faster synchronization with a low amplitude of oscillations of the error signals compared to the controllers reported in [17, 27].

\begin{tabular}{|c|c|c|c|}
\hline $\begin{array}{c}\text { NLC } \\
\text { controller }\end{array}$ & $\begin{array}{l}\text { Controller } \\
\text { parameters }\end{array}$ & $\begin{array}{c}\text { Convergence } \\
\text { time }\end{array}$ & $\begin{array}{l}\text { Closed-loop system } \\
\text { oscillation interval }\end{array}$ \\
\hline $\begin{array}{c}u(t) \\
\text { (Proposed) }\end{array}$ & $\begin{array}{c}\mathrm{K}=500[1 ; 1 ; 0.05] \\
\rho=[500 ; 10 ; 10] \\
\gamma=0.1 \\
\mathrm{~L}=20\end{array}$ & $\mathrm{t}=0.2 \mathrm{~s}$ & {$[-2,0.2]$} \\
\hline$u(t)$ [17] & $\begin{array}{c}\beta=0.75 \\
\mathrm{~K}=29(0.8)-1\end{array}$ & $\mathrm{t}=0.4 \mathrm{~s}$ & {$[-2,1.4]$} \\
\hline$u(t)$ [27] & $\begin{array}{c}\mathrm{K}=20 \\
\beta_{1}=(25 \alpha+10)>0 \\
\beta_{2}=28-35 \alpha \\
\beta_{3}=29 \alpha+1\end{array}$ & $\mathrm{t}=1.5 \mathrm{~s}$ & {$[-2,1.3]$} \\
\hline
\end{tabular}

Table 2: Comparative analysis of the simulation results.

Figure 4 (a)-(c) show the control inputs of the proposed controller are oscillation free, while the controllers in $[17,27]$ synthesize oscillatory control signals. The oscillatory error synchronization behavior is the consequence of these oscillations. The large amplitude oscillation in the input signals increases the time of convergence and use more energy. 


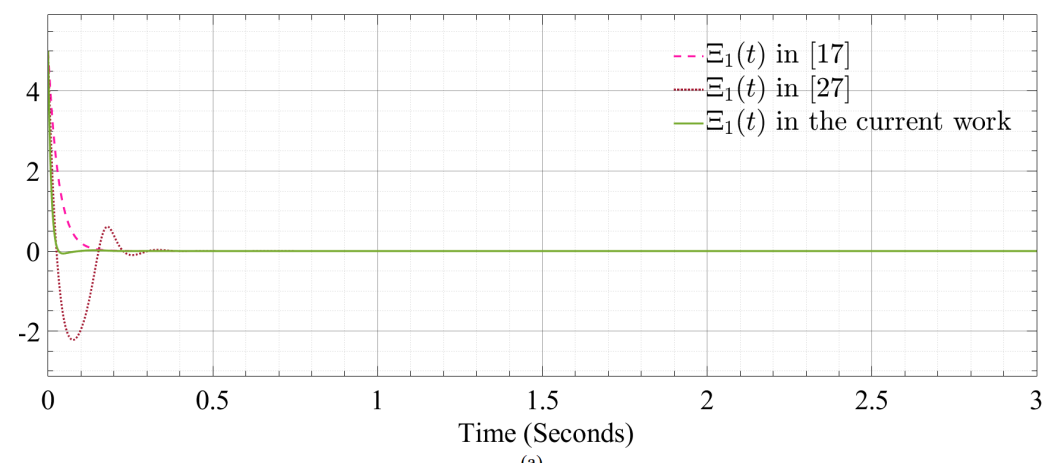

(a)

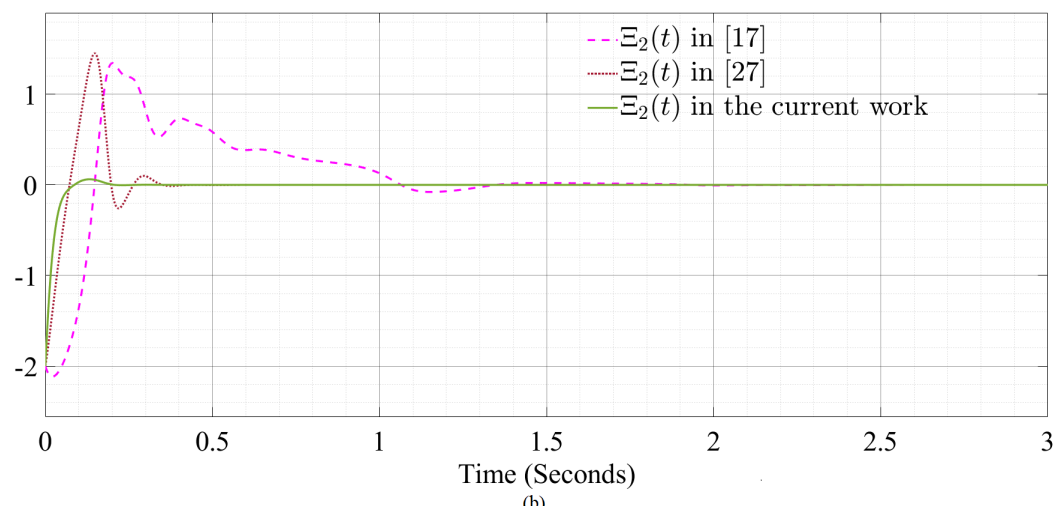

(b)

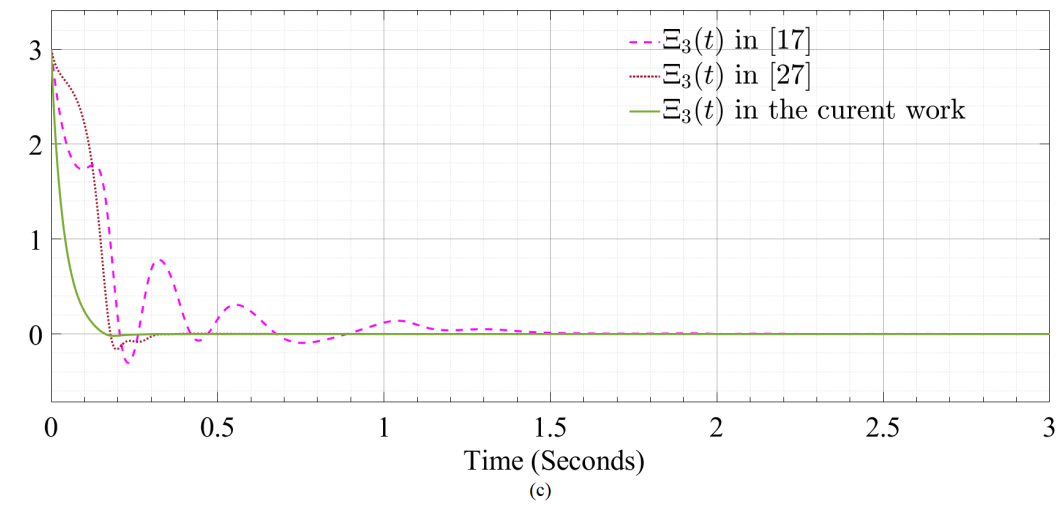

Figure 3: Transient behavior of the error signal, (a) $\Xi_{1}(t),(b) \Xi_{2}(t)$, and (c) $\Xi_{3}(t)$.

\section{Numerical example-2}

This section illustrates the synchronization between two identical hyperchaotic Lu systems.

\subsection{Dynamics of the hyperchaotic Lu system}

Equation (5.1) demonstrates the dynamics of the hyperchaotic Lu system.

$$
\left\{\begin{array}{l}
\dot{\chi}_{1}(t)=a\left(\chi_{2}(t)-\chi_{1}(t)\right)+\chi_{4}(t) \\
\dot{\chi}_{2}(t)=-\chi_{1}(t) \chi_{3}(t)+b x_{2}(t) \\
\dot{\chi}_{3}(t)=x_{1}(t) \chi_{2}(t)-c x_{3}(t) \\
\dot{\chi}_{4}(t)=x_{1}(t) \chi_{3}(t)+d \chi_{4}(t)
\end{array}\right.
$$

where $\chi_{1}(t), \chi_{2}(t), \chi_{3}(t)$, and $\chi_{4}(t)$ are the state-variable, and $a=36, b=3, c=20$, and $d=-0.35 \leqslant$ $\rho \leqslant 1.2$ are the parameters. Figure 5 depicts the behavior of the state variables, and Figure 6 (a-d) shows the phase portraits of the hyperchaotic system (5.1). 


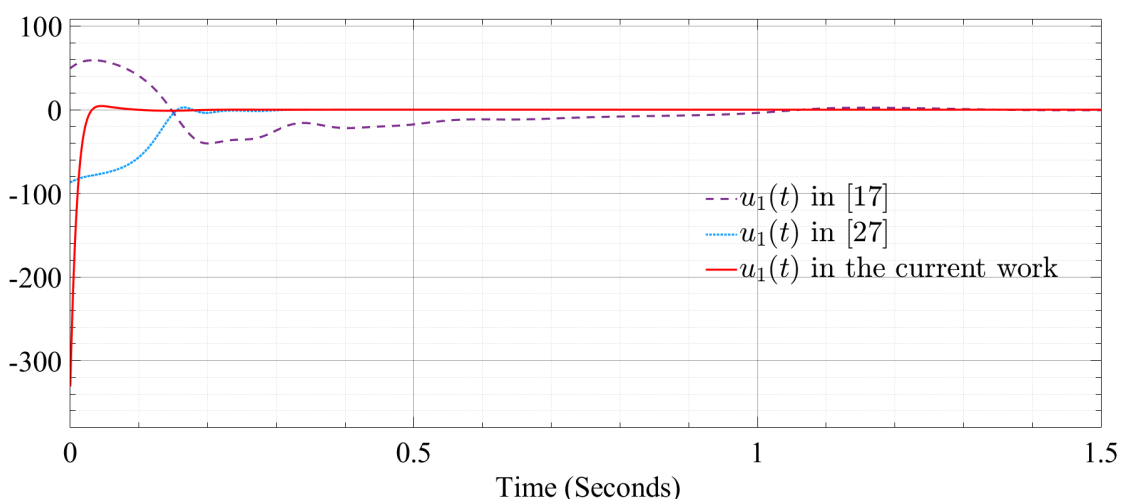

(a)

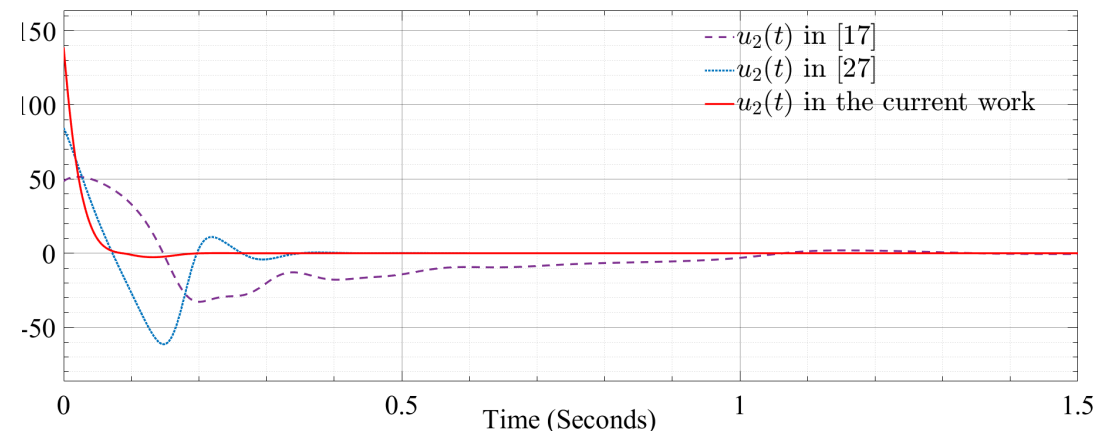

(b)

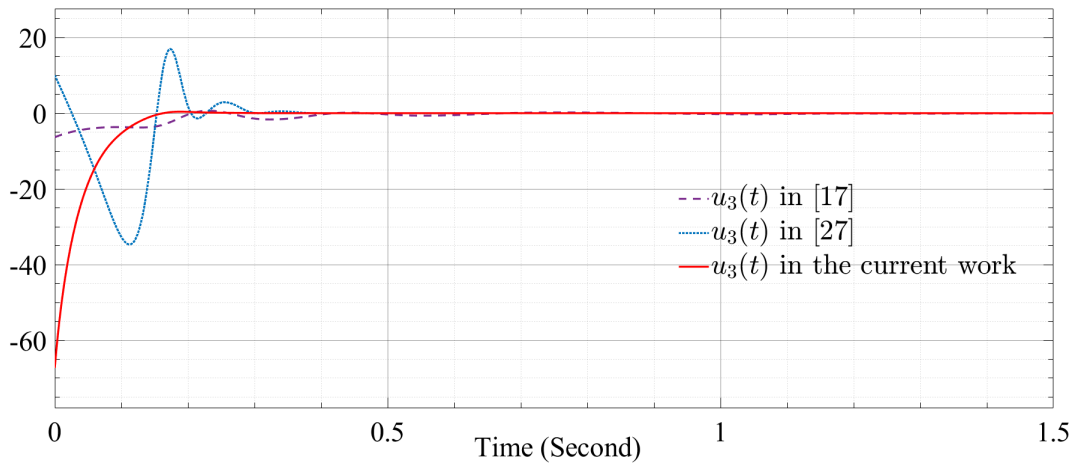

(c)

Figure 4: Comparative performance of the control inputs, (a) $u_{1}(t),(b) u_{2}(t)$, and $(c) u_{3}(t)$.

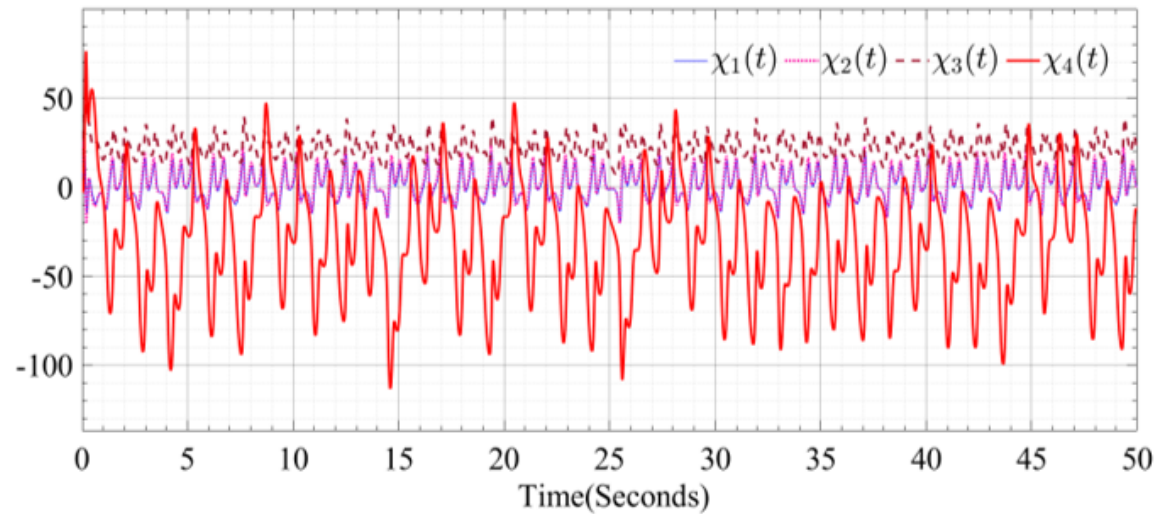

Figure 5: Behavior of the state variables of the hyperchaotic Lu system. 


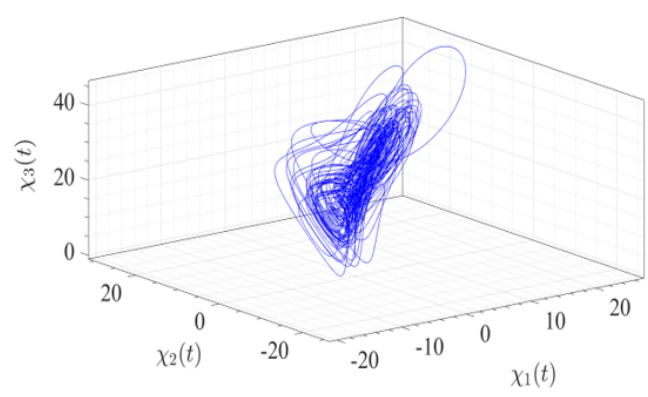

(a)

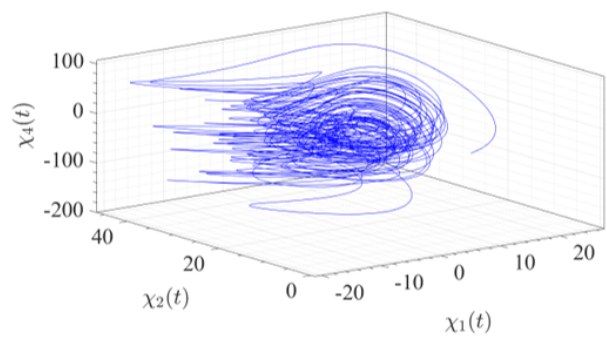

(c)

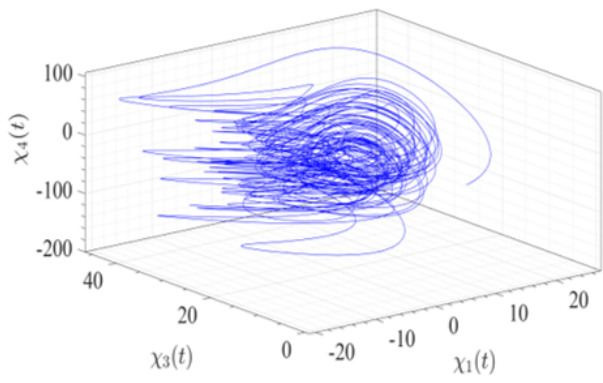

(b)

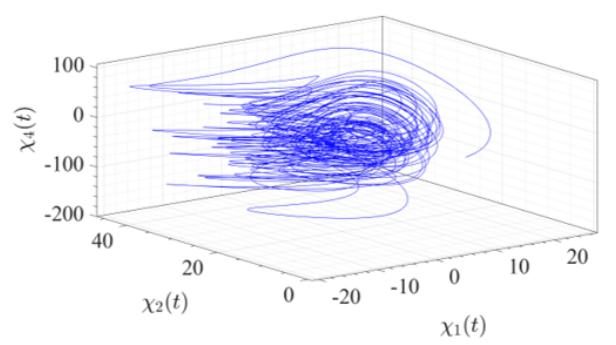

(d)

Figure 6: 3D phase portraits of the hyperchaotic Lu system, (a) $\chi_{1}(t), \chi_{2}(t), \chi_{3}(t),(b) \chi_{1}(t), \chi_{3}(t), \chi_{4}(t)(c) \chi_{1}(t), \chi_{2}(t), \chi_{4}(t)$, and $\left.(d) \chi_{2}(t), \chi_{3}(t), \chi_{4}(t)\right)$.

\subsection{Synchronization of two identical hyperchaotic Lu systems}

Consider a master-slave system arrangement given in (5.2) and (5.3). The state vector of the master system is denoted by $\chi^{m}(t)=\left[\chi_{1}^{m}(t), \chi_{2}^{m}(t), \chi_{3}^{m}(t), \chi_{4}^{m}(t)\right]$, and the slave system is represented by $\chi^{s}(t)=\left[\chi_{1}^{s}(t), \chi_{2}^{s}(t), \chi_{3}^{s}(t), \chi_{4}^{s}(t)\right]$ with the feedback control input $u(t)=\left[u_{1}(t), u_{2}(t), u_{3}(t), u_{4}(t)\right]$.

$$
\begin{aligned}
& \text { Master system: }\left\{\begin{array}{l}
\dot{\chi}_{1}^{m}(t)=a\left(\chi_{2}^{m}(t)-\chi_{1}^{m}(t)\right)+\chi_{4}^{m}(t), \\
\dot{\chi}_{2}^{m}(t)=-\chi_{1}^{m}(t) x_{3}^{m}(t)+b \chi_{2}^{m}(t), \\
\dot{\chi}_{3}^{m}(t)=x_{1}^{m}(t) \chi_{2}^{m}(t)-c x_{3}^{m}(t), \\
\dot{\chi}_{4}^{m}(t)=\chi_{1}^{m} \chi_{3}^{m}(t)+d x_{4}^{m}(t),
\end{array}\right. \\
& \text { Slave system : }\left\{\begin{array}{l}
\dot{\chi}_{1}^{s}(t)=a\left(\chi_{2}^{s}(t)-\chi_{1}^{s}(t)\right)+\chi_{4}^{s}(t)+u_{1}(t), \\
\dot{\chi}_{2}^{s}(t)=-\chi_{1}^{s}(t) \chi_{3}^{s}(t)+b \chi_{2}^{s}(t)+u_{2}(t), \\
\dot{\chi}_{3}^{s}(t)=\chi_{1}^{s}(t) \chi_{2}^{s}(t)-c \chi_{3}^{s}(t)+u_{3}(t), \\
\dot{\chi}_{4}^{s}(t)=\chi_{1}^{s}(t) \chi_{3}^{s}(t)+d \chi_{4}^{s}(t)+u_{4}(t) .
\end{array}\right.
\end{aligned}
$$

The error dynamics in (5.4) is the difference of (5.2) and (5.3),

$$
\left\{\begin{array}{l}
\dot{\Xi}_{1}(t)=a\left(\Xi_{2}(t)-\Xi_{1}(t)\right)+\Xi_{4}(t)+u_{1}(t), \\
\dot{\Xi}_{2}(t)=-x_{1}^{s}(t) \chi_{3}^{s}(t)+\chi_{1}^{m}(t) \chi_{3}^{m}(t)+b \Xi_{2}(t)+u_{2}(t), \\
\dot{\Xi}_{3}(t)=x_{1}^{s}(t) \chi_{2}^{s}(t)-\chi_{1}^{m}(t) \chi_{2}^{m}(t)-c \Xi_{3}(t)+u_{3}(t), \\
\dot{\Xi}_{4}(t)=x_{1}^{s}(t) \chi_{3}^{s}(t)-x_{1}^{m}(t) x_{3}^{m}(t)+d \Xi_{4}+u_{4}(t) .
\end{array}\right.
$$

Substituting (4.5) in (5.4) gives (5.5),

$$
\left\{\begin{array}{l}
\dot{\Xi}_{1}(\mathrm{t})=\mathrm{a}\left(\Xi_{2}(\mathrm{t})-\Xi_{1}(\mathrm{t})\right)+\Xi_{4}(\mathrm{t})+\mathrm{u}_{1}(\mathrm{t}), \\
\dot{\Xi}_{2}(\mathrm{t})=-x_{3}^{\mathrm{s}}(\mathrm{t}) \Xi_{1}(\mathrm{t})-x_{1}^{\mathrm{s}}(\mathrm{t}) \Xi_{3}(\mathrm{t})+\Xi_{1}(\mathrm{t}) \Xi_{3}(\mathrm{t})+\mathrm{b} \Xi_{2}(\mathrm{t})+\mathrm{u}_{2}(\mathrm{t}), \\
\dot{\Xi}_{3}(\mathrm{t})=x_{2}^{\mathrm{s}}(\mathrm{t}) \Xi_{1}(\mathrm{t})+x_{1}^{\mathrm{s}}(\mathrm{t}) \Xi_{2}-\Xi_{1}(\mathrm{t}) \Xi_{2}(\mathrm{t})-\mathrm{c} \Xi_{3}(\mathrm{t})+\mathrm{u}_{3}(\mathrm{t}), \\
\dot{\Xi}_{4}(\mathrm{t})=x_{3}^{\mathrm{s}}(\mathrm{t}) \Xi_{1}(\mathrm{t})+x_{1}^{\mathrm{s}}(\mathrm{t}) \Xi_{3}(\mathrm{t})-\Xi_{1}(\mathrm{t}) \Xi_{3}(\mathrm{t})+\mathrm{d} \Xi_{4}(\mathrm{t})+\mathrm{u}_{4}(\mathrm{t}) .
\end{array}\right.
$$

The control signal in (3.1) globally asymptotically stabilizes the error dynamical system (5.5) at the origin. 


\subsection{Numerical simulations and comparative study}

This subsection demonstrates the performance of the control law (3.2) in terms of synchronization error to zero, and time of convergence and describes the comparative analysis with the feedback controller [12].

The initial conditions for the master and slave hyperchaotic Lu systems are chosen as

$$
\left[\chi_{1}^{m}(0), \chi_{2}^{m}(0), \chi_{3}^{m}(0), \chi_{4}^{m}(0)\right]^{\top}=[5,8,-1,-3]^{\top} \text { and }\left[\chi_{1}^{s}(0), \chi_{2}^{s}(0), \chi_{3}^{s}(0), \chi_{4}^{s}(0)\right]^{\top}=[3,4,5,5]^{\top} \text {, }
$$

respectively. The feedback gains $K$ and $L$ are such that $K=\operatorname{diag}[500 ; 500 ; 25 ; 25]$ and $L=\operatorname{diag}[20 ; 20 ; 20 ; 20]$. The controller parameters $\rho$ and $\gamma$ are set as $\rho=0.01$ and $\gamma=0.1$.

The control signals $u_{i}(t), i=1,2,3,4$, given in (5.6) are synthesized by the feedback control strategy [12], then these control signals in the error system (5.5) establish the synchronization of the master and slave systems (5.2) and (5.3).

The feedback control technique [12]:

$$
\left\{\begin{array}{l}
\mathrm{u}_{1}(\mathrm{t})=-\alpha \Xi_{2}(\mathrm{t})-\Xi_{4}(\mathrm{t}), \\
\mathrm{u}_{2}(\mathrm{t})=\chi_{3}^{\mathrm{s}}(\mathrm{t}) \Xi_{1}(\mathrm{t})+\chi_{1}^{s}(\mathrm{t}) \Xi_{3}(\mathrm{t})-\Xi_{1}(\mathrm{t}) \Xi_{3}(\mathrm{t})-\beta \Xi_{2}(\mathrm{t})-\Xi_{2}(\mathrm{t}), \\
\mathrm{u}_{3}(\mathrm{t})=-\chi_{2}^{s}(\mathrm{t}) \Xi_{1}(\mathrm{t})-\chi_{1}^{s}(\mathrm{t}) \Xi_{2}+\Xi_{1}(\mathrm{t}) \Xi_{2}(\mathrm{t}), \\
\mathrm{u}_{4}(\mathrm{t})=-\chi_{3}^{s}(\mathrm{t}) \Xi_{1}(\mathrm{t})-\chi_{1}^{s}(\mathrm{t}) \Xi_{3}(\mathrm{t})+\Xi_{1}(\mathrm{t}) \Xi_{3}(\mathrm{t})-\rho \Xi_{4}(\mathrm{t})-\Xi_{4}(\mathrm{t})
\end{array}\right.
$$

Figures 7 and 8 illustrate the transient behavior of the errors in the current work and by [12], respectively. Figures 7 and 8 illustrate that the current work accomplishes the synchronization behavior after $\mathrm{t}=0.3$ seconds, while the controller in [12] achieves the synchronization behavior after $\mathrm{t}=4$ seconds. Fast synchronization is vital in secure communication; it improves the response time that reduces the hacking risk during the data encryption W9988W10210.

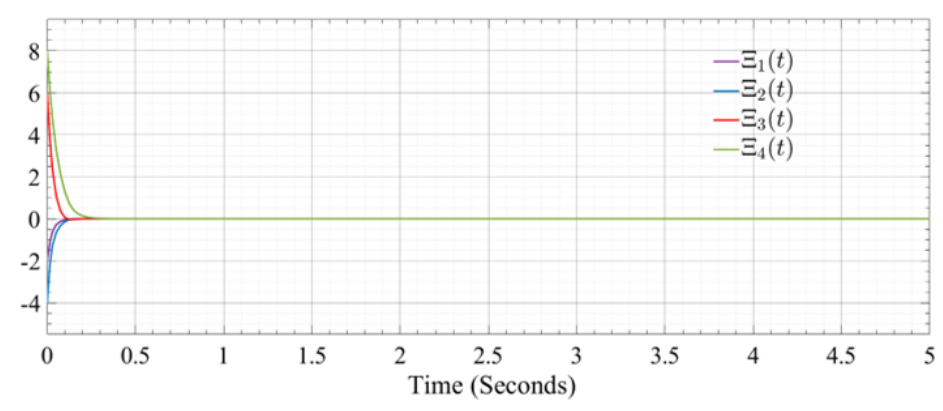

Figure 7: Transient behavior of the errors in the current work.

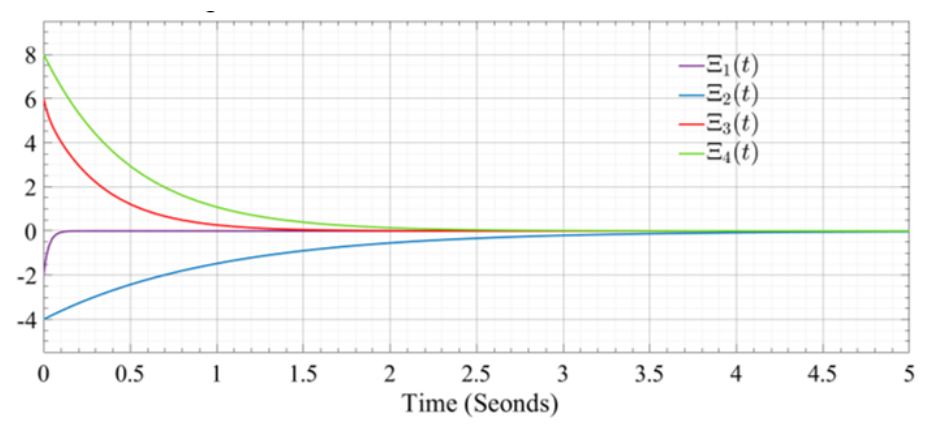

Figure 8: Transient behavior of the errors in [12]. 


\section{Conclusions}

This paper proposes a novel nonlinear feedback controller that accomplishes fast and oscillation free convergence of the synchronization errors to zero. The paper describes a detailed mathematical analysis of the proposed nonlinear control strategy, which shows that the synchronization error is oscillation free, and the time of convergence is less. The Lyapunov second theorem proves the global asymptotic stability of the closed-loop system at the origin. The paper also discusses computer simulation results and compare them with state-of-the-art controllers.

\section{References}

[1] I. Ahmad, A. B. Saaban, A. B. Ibrahim, M. Shahzad, N. Naveed, The Synchronization of Chaotic Systems with Different Dimensions by a Robust Generalized Active Control, Optik, 127 (2016), 4859-4871. 1

[2] I. Ahmad, M. Shafiq, Robust adaptive anti-synchronization control of multiple uncertain chaotic systems of different orders, Automatika, 61 (2020), 396-414. 1

[3] I. Ahmad, M. Shafiq, Oscillation free robust adaptive synchronization of chaotic systems with parametric uncertainties, Trans. Inst. Meas. Control., 42 (2020), 1977-1996. 1

[4] I. Ahmad, M. Shafiq, M. M. Al-Sawalha, Globally exponential multi switching-combination synchronization control of chaotic systems for secure communications, Chin. J. Phys., 56 (2018), 974-987. 1

[5] I. Ahmad, M. Shafiq, A. B. Saaban, A. B. Ibrahim, M. Shahzad, Robust finite-time global synchronization of chaotic systems with different orders, Optik, 127 (2016), 8172-8185. 1

[6] M. M. Al-sawalha, A. F. Al-Dababseh, Nonlinear Anti-Synchronization of Two Hyper chaotic Systems, Appl. Math. Sci., 5 (2011), 1849-1856. 1

[7] A. Biswas, R. Fujimoto,Energy consumption of synchronization algorithms in distributed simulations, J. Simul., 11 (2017), 242-252. 1, 3.1

[8] G. Cai, Z. Tan, Chaos synchronization of a new chaotic system via nonlinear control, J. Uncertain Syst., 1 (2007), $235-240$. 1

[9] X. Chen, J. H. Park, J. Cao, J. Qiu, Sliding mode synchronization of multiple chaotic systems with uncertainties and disturbances, Appl. Math. Comput., 308 (2017), 161-173. 1

[10] A. Dinesh, B. B. Sharma, Synchronization of Two Different Chaotic Systems in the Presence of External Disturbances, 2018 Second International Conference on Electronics, Communication and Aerospace Technology (ICECA), IEEE, (2018). 1

[11] L. Huang, R. Feng, M. Wang, Synchronization of chaotic systems via nonlinear control, Phys. Lett. A, 320 (2004), 271-275. 1

[12] A. Khan, S. Singh Chaotic analysis and combination-combination synchronization of a novel hyperchaotic system without any equilibria, Chin. J. Phys., 56 (2018), 238-251. 5.3, 5.3, 8

[13] H. Kheiri, B. Naderi, Dynamical behavior and synchronization of chaotic chemical reactors model, Iranian J. Math. Chem., 6 (2015), 75-86. 1

[14] X.-F. Li, Y.-D. Chu, A. Y. T. Leung, H. Zhang, Synchronization of uncertain chaotic systems via complete-adaptiveimpulsive controls, Chaos Soliton. Fract., 100 (2017), 24-30. 1

[15] W.-L. Li, W.-L. Liang, K.-M. Chang, Adaptive Sliding Mode Control for Synchronization of Unified Hyperchaotic Systems, In 2019 24th International Conference on Methods and Models in Automation and Robotics (MMAR), IEEE, (2019), 93-98. 1

[16] M. Maheri, N. M. Arifin, Application adaptive exponential synchronization of chaotic dynamical systems in secure communications, Adv. Difference Equ., 2017 (2017), 21pages. 1

[17] J. H. Park, On synchronization of unified chaotic systems via nonlinear control, Chaos Soliton. Fract., 25 (2005), 699-704. $1,4.3,4.3,2,4.3$

[18] L. M. Pecora, T. L. Carroll, Synchronization in chaotic systems, Phys. Rev. Lett., 64 (1990), 821-824. 1

[19] M. H. Sabzalian, A. Mohammadzadeh, S. Lin, W. Zhang, Robust fuzzy control for fractional-order systems with estimated fraction-order, Nonlinear Dyn., 98 (2019), 2375-2385. 1

[20] M. Shafiq, I. Ahmad, Multi-switching combination anti-synchronization of unknown hyperchaotic systems, Arab. J. Sci. Eng., 44 (2019), 7335-7350. 1

[21] M. Shahzad, A. B. Saaban, A. B. Ibrahim, I. Ahmad, Adaptive control to synchronize and anti-synchronize two identical time delay Bhalekar-Gejji chaotic systems with unknown parameters, Int. J. Autom. Control., 9 (2015), 211-227. 1

[22] M. K. Shukla, B. B. Sharma, Stabilization of a class of fractional order chaotic systems via backstepping approach, Chaos Soliton. Fract., 98 (2017), 56-62. 1

[23] J. Sun, Y. Wang, Y. Wang, Y. Shin, Finite-time synchronization between two complex-variable chaotic systems with unknown parameters via nonsingular terminal sliding mode contro, Nonlinear Dyn., 85 (2016), 1105-1117. 1

[24] P. P. Singh, J. P. Singh, B. K. Roy, Synchronization and anti-synchronization of Lu and Bhalekar-Gejji chaotic systems using nonlinear active control, Chaos Soliton. Fract., 69 (2014), 31-39. 1 
[25] S. Singh, Single input sliding mode control for hyperchaotic Lu system with parameter uncertainty,Int. J. Dyn. Control., 4 (2016), 504-514. 1

[26] L. Wang, T. Chen, Finite-time anti-synchronization of neural networks with time-varying delays, Neurocomputing, 275 (2018), 1595-1600. 1

[27] H. Wang. Z.-Z. Han, Q.-Y. Xie, W. Zhang, Finite-time chaos synchronization of unified chaotic system with uncertain parameters, Commun. Nonlinear Sci. Numer. Simul., 14 (2009), 2239-2247. 1, 4.3, 4.3, 4.3, 2, 4.3

[28] Y. Wang , H. Yu, Fuzzy synchronization of chaotic systems via intermittent control, Chaos Soliton. Fract., 106 (2018), 154-160. 1

[29] X. Yang, Q. Song, J. Cao, J. Lu, Synchronization of coupled Markovian reaction-diffusion neural networks with proportional delays via quantized control, IEEE Trans. Neural Netw. Learn. Syst., 30 (2019), 951-958. 1

[30] Q. Zhang, J.-A. Lu, Chaos synchronization of a new chaotic system via nonlinear control, Chaos Soliton. Fract., 37 (2008), 175-179. 